Review began 02/04/2022 Review ended 02/19/2022 Published 02/21/2022

(๑) Copyright 2022

Sindhlian et al. This is an open access article distributed under the terms of the Creative Commons Attribution License CCBY 4.0., which permits unrestricted use, distribution, and reproduction in any medium, provided the original author and source are credited.

\section{Sepsis in Critically Ill Patients: Epidemiology, Risk Factors, and Role of Matrix-Assisted Laser Desorption/Ionization-Time of Flight Mass Spectrometry for Identification of Sepsis-Causing Organisms}

Kuldeep Singh Sindhlian ${ }^{1}$, Zia Arshad ${ }^{2}$, Prashant Gupta ${ }^{3}$, Vipin K. Singh ${ }^{1}$, Gyan Prakash Singh ${ }^{1}$, Haider Abbas 4

1. Department of Anaesthesiology, King George's Medical University, Lucknow, IND 2. Department of Anaesthesia and Critical Care, King George's Medical University, Lucknow, IND 3. Department of Mircrobiology, King George's Medical University, Lucknow, IND 4. Department of Emergency Medicine, King George's Medical University, Lucknow, IND

Corresponding author: Zia Arshad, ziaarshad13@gmail.com

\section{Abstract}

\section{Background}

Matrix-assisted laser desorption/ionization-time of flight mass spectrometry (MALDI-TOF MS) is a rapid mass spectrometry technology for species identification. It is a useful, fast, and accurate tool for routine laboratory analysis. This study aimed to investigate the epidemiology of sepsis-causing organisms in patients admitted to tertiary-level intensive care units (ICU), the role of MALDI-TOF MS in species identification, and patients' clinical outcomes.

\section{Methodology}

A prospective observational study was performed in a tertiary-level ICU for one year. The first blood, urine, and endotracheal (ET) aspirate samples were sent before starting antibiotics. We received the antibiotic sensitivity report within 48 hours or earlier using MALDI-TOF MS. Treatment was modified based on MALDI-TOF MS reports. Response to treatment was monitored, and clinical outcomes were noted based on ICU stay. Patients were followed up until discharge, shifting to referring parent unit, or death.

\section{Results}

This study included 200 patients admitted to ICUs who at the time of admission did not have a fever. The most common organisms were Acinetobacter baumannii, Klebsiella pneumonia, and Escherichia coli in ET aspirates; Candida albicans and Enterococcus faecium in urine; and Pseudomonas aeruginosa, K. pneumonia, and A. baumanii in blood. Of the 200 patients, 130 (65\%) shifted to the parent unit ward, and 70 (35\%) patients died, with an ICU stay of $12.89 \pm 6.51$ days. There was no significant difference in mortality when organisms grew from either ET or urine compared with sterile samples. If organisms resistant to all primary antibiotics grew from ET, mortality was $60.6 \%$. Mortality was $56.8 \%$ if isolates were in the blood.

\section{Conclusions}

Early MALDI-TOF MS-based species identification and appropriate antibiotics initiation play a key role in the treatment and care for critically ill patients with sepsis. MALDI-TOF MS has the potential to significantly aid sepsis management.

Categories: Anesthesiology, Internal Medicine, Integrative/Complementary Medicine

Keywords: antibiotics, sepsis-causing organism, maldi-tof ms, intensive care unit, sepsis

\section{Introduction}

Matrix-assisted laser desorption/ionization-time of flight mass spectrometry (MALDI-TOF MS) is a rapid mass spectrometry technology developed in the late 1980s. It is a useful, fast, and accurate tool for routine laboratory analysis. Several studies have reported that the introduction of MALDI-TOF MS reduces intensive care unit (ICU) and hospital stays of patients with bacteremia and/or candidemia and markedly decreases hospital costs $[1,2]$. However, to our knowledge, the impact of MALDI-TOF MS on the clinical outcomes of patients with sepsis, especially critically ill patients requiring admission to an ICU, remains poorly understood. MALDI-TOF MS utilization in a clinical microbiology laboratory has markedly increased over the past 10 years [3]. Over time, platforms have progressively advanced, with significant improvements in the software, interpretive rules, and databases. Consequently, there is limited value in comparing results for any category of organisms using a retrospective literature review. In this study, we hypothesized that rapid 
MALDI-TOF MS-based identification on top of a well-established antimicrobial stewardship program (ASP) would significantly improve antibiotic management compared with an ASP using conventional identification even in a hospital setting with a low prevalence of resistant organisms. Consequently, we conducted this prospective study of a one-year duration investigating the epidemiology, risk factors, and role of MALDITOF MS in species identification of sepsis-causing organisms.

This study aimed to investigate the epidemiology of sepsis-causing organisms in patients admitted to tertiary-level ICU care, the role of MALDI-TOF MS in species identification, and patients' clinical outcomes.

\section{Materials And Methods}

\section{Study setting and design}

This prospective observational study was conducted in a tertiary-level ICU from September 2019 to August 2020. In this study, we performed convenience non-probability sampling. The sample size was determined using the following equation: $N=(r+1 / r) \times \sigma^{2}(Z \beta+Z \alpha / 2)^{2} /(\text { difference })^{2}$, where $n$ is the number needed in each group, $r$ is the ratio of control to cases (valued at 1 , an equal number of cases and control), $\sigma$ is the standard deviation from the reference study, $Z \beta$ is the standard normal variate for a set power $(0.84$ for $80 \%$ power), $Z \alpha / 2$ is the standard normal variate for the level of significance (1.96 for $95 \%$ confidence interval, $\mathrm{CI})$, and the difference of means in the reference study $(\mathrm{d}=0.7)$ was 196.85 . Finally, 200 cases included in the study. The minimum sample size for this study was statistically calculated based on the study by Chatterjee et al. [4].

\section{Inclusion and exclusion criteria}

We included patients aged 16-60 years who were admitted to the ICU, those with new-onset fever after 48 hours of hospital admission, and those with new-onset neutropenia with fever. Because the study was conducted in adult ICU, pediatrics patients were excluded. As the number of patients above the age of 60 years was low, we included patients between the age group of 16 and 60 years.

We excluded patients unwilling to provide consent, those having fever at admission, and those developing fever within 48 hours of admission. Additionally, we excluded all patients who presented with the diagnosis of sepsis at the time of admission.

\section{Study procedure}

The study was performed in a tertiary-level ICU. If a patient met the inclusion criteria and was willing to participate, informed consent was obtained. The first sample was collected at the time of admission. Repeat samples after developing fever with two sets of peripheral blood cultures were collected, each containing at least 8-10 mL. One sample from the central venous line was also collected (if applicable) along with peripheral blood culture. We received an antibiotic sensitivity report within 48 hours or earlier using MALDITOF MS. Treatment was initiated based on MALDI-TOF MS and susceptibility test findings. Response to treatment was monitored, and clinical outcomes were documented based on the length of ICU stay. The cases were followed up till the patients were discharged, shifted to a referring parent unit ward, or deceased.

\section{Ethical approval}

Ethical approval was obtained from the Institutional Ethics Committee (King George's Medical University, Lucknow, India; DCGI registration number: ECR/262/inst/up/2013/RR-19), with approval number 102 ECM II B-THESIS/P79.

\section{Statistical analysis}

The data were entered in Microsoft Excel and analyzed using SPSS version 23 (IBM Corp., Armonk, NY USA). The Student's t-test was used to analyze parametric data, and the Mann-Whitney U test was used to analyze non-parametric data. Fisher's test was used to analyze categorical data. The confidence interval (CI) was set at $95 \%$, the alpha value was 0.05 , the power of $1-ß$ was 0.8 , and the level of statistical significance was set at P-values of $<0.05$.

\section{Results}

In this study, the most common isolated organisms were Acinetobacter baumannii, Klebsiella pneumoniae, Escherichia coli, and Pseudomonas aeruginosa in endotracheal (ET) aspirates; Candida albicans, Enterococcus faecium, and C. glabrata in urine; and P. aeruginosa, A. baumannii, and K. pneumoniae in the blood (Table 1). This study was conducted in a government-aided hospital where we received referred patients with sepsis-induced multiorgan dysfunction syndrome (MODS) with multidrug-resistant infections. Crossinfection may be one of the causes for the high incidence of multidrug-resistant organisms. Because we only included patients who developed a fever after admission, it may be one of the causes of high incidence. 


\section{Cureus}

\begin{tabular}{|c|c|c|c|}
\hline & Site $(N=200)$ & Organism & Numbers $(\mathrm{N})$ \\
\hline \multirow{7}{*}{ Sample 1} & \multirow{7}{*}{ ETA $(N=186)$} & Acinetobacter baumannii & 91 \\
\hline & & Klebsiella pneumoniae & 14 \\
\hline & & Escherichia coli & 9 \\
\hline & & Pseudomonas aeruginosa & 8 \\
\hline & & Proteus mirabilis & 3 \\
\hline & & Staphylococcus aureus & 3 \\
\hline & & Sterile & 58 \\
\hline \multirow{12}{*}{ Sample 2} & Non-intubated patients $(n=14)$ & & 14 \\
\hline & \multirow{10}{*}{ Urine $(N=194)$} & Candida albicans & 10 \\
\hline & & Enterococcus faecium & 9 \\
\hline & & Candida glabrata & 6 \\
\hline & & Enterococcus faecalis & 6 \\
\hline & & Escherichia coli & 3 \\
\hline & & Candida auris & 3 \\
\hline & & Klebsiella pneumoniae & 3 \\
\hline & & Candida orthopsilosis & 3 \\
\hline & & Trichosporon asahii & 3 \\
\hline & & Sterile & 137 \\
\hline & Sample not sent $(\mathrm{N}=6)$ & & 6 \\
\hline \multirow{8}{*}{ Sample 3} & \multirow{7}{*}{ Blood (N = 197) } & Acinetobacter baumannii & 9 \\
\hline & & Pseudomonas aeruginosa & 10 \\
\hline & & Klesbiella pneumoniae & 9 \\
\hline & & Stenotrophomonas maltophilia & 3 \\
\hline & & Enterococcus faecalis & 4 \\
\hline & & Staphylococcus aureus & 2 \\
\hline & & Sterile & 160 \\
\hline & Sample not sent $(\mathrm{N}=3)$ & & 3 \\
\hline \multirow{7}{*}{ Miscellaneous } & Drain $(\mathrm{N}=2)$ & Escherichia coli & 2 \\
\hline & Placenta membrane $(\mathrm{N}=3)$ & Escherichia coli & 3 \\
\hline & \multirow{4}{*}{ Pus $(\mathrm{N}=10)$} & Escherichia coli & 6 \\
\hline & & Klebsiella pneumoniae & 3 \\
\hline & & Pseudomonas aeruginosa & 2 \\
\hline & & Staphylococcus epidermidis & 2 \\
\hline & Sputum $(\mathrm{N}=2)$ & Acinetobacter baumannii & 2 \\
\hline
\end{tabular}

TABLE 1: Distribution of organisms from various samples.

ETA: endotracheal aspirate 


\section{Cureus}

In the study, $10 \%$ of the studied patients had diabetes, followed by hypertension ( $7.5 \%)$, chronic obstructive pulmonary disease (4.5\%), severe acute respiratory syndrome coronavirus 2 (SARS-CoV-2)

infection (coronavirus disease 2019, COVID-19) (3.5\%), and hepatitis C virus (HCV) infection (3.0\%), whereas $72.0 \%$ did not have any of the above risk factors (Figure 1).

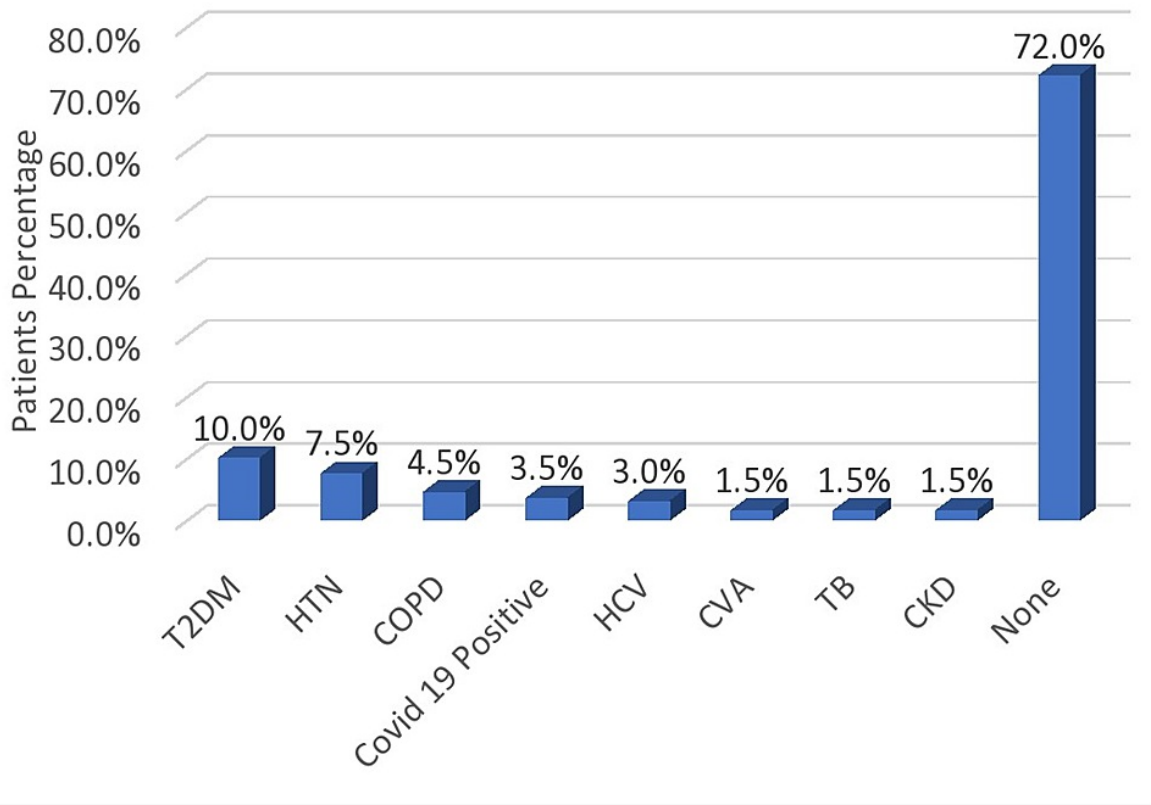

\section{FIGURE 1: Comorbidities seen in the study population.}

T2DM: type 2 diabetes mellitus; HTN: hypertension; COPD: chronic obstructive pulmonary disease; COVID-19: coronavirus disease 2019; HCV: hepatitis C virus; CVA: cerebrovascular accident; TB: tuberculosis; CKD: chronic kidney disease

In 2018 , mortality was $35.36 \%$ (325/919), and in 2019, it was $35.34 \%$ (328/928). Thus, in our study, mortality was approximately 35\%. Data were collected from the institutional records maintained in the ICU (Table 2).

\begin{tabular}{|c|c|c|c|}
\hline Year & Total patients & Mortality & Percentage \\
\hline 2018 & 919 & 325 & $35.36 \%$ \\
\hline 2019 & 928 & 328 & $35.34 \%$ \\
\hline 2020 (present study) & 200 & 70 & $35.0 \%$ \\
\hline
\end{tabular}

TABLE 2: Year-wise mortality.

Based on the antibiotics susceptibility tests of organisms isolated from ET aspirates, A. baumanii was most susceptible to tetracycline, tobramycin, levofloxacin, and meropenem. In blood, $P$. aeuroginosa was the most common isolated organism and was most susceptible to piperacillin/tazobactam and gentamycin. In urine, Candida spp. was susceptible to both fluconazole and voriconazole (Tables 3, 4). Patients who developed symptoms or neutropenia were treated with fluconazole. 


\section{Cureus}

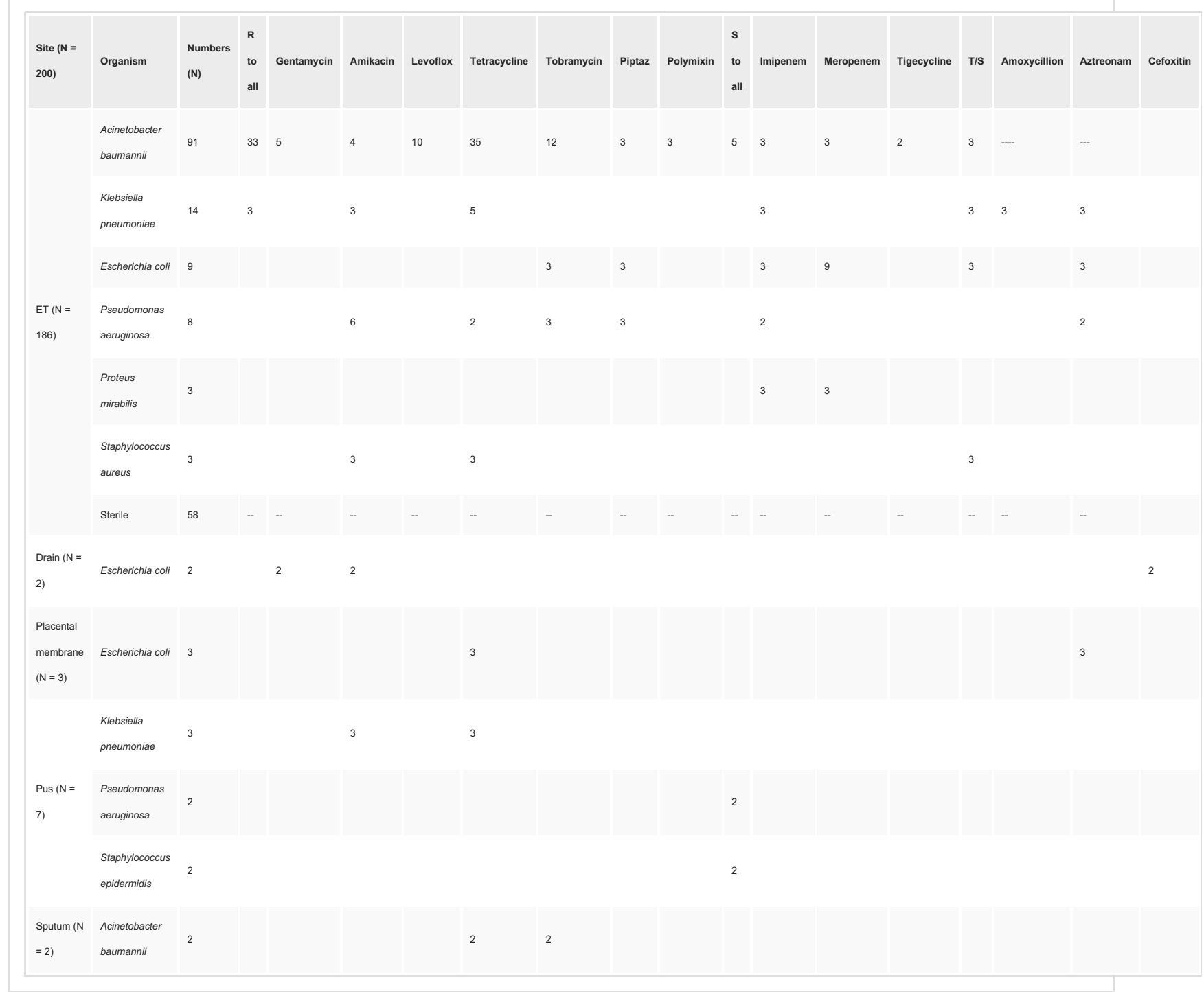

TABLE 3: Antibiotic susceptibility pattern of the organisms in ET and miscellaneous causing septicemia.

$\mathrm{R}$ to all: resistant to all primary antibiotics; $\mathrm{S}$ to all: sensitive to all primary antibiotics, Piptaz: piperacillin/tazobactam; T/S: trimethoprim/sulfamethoxazole; ET: endotracheal 


\section{Cureus}

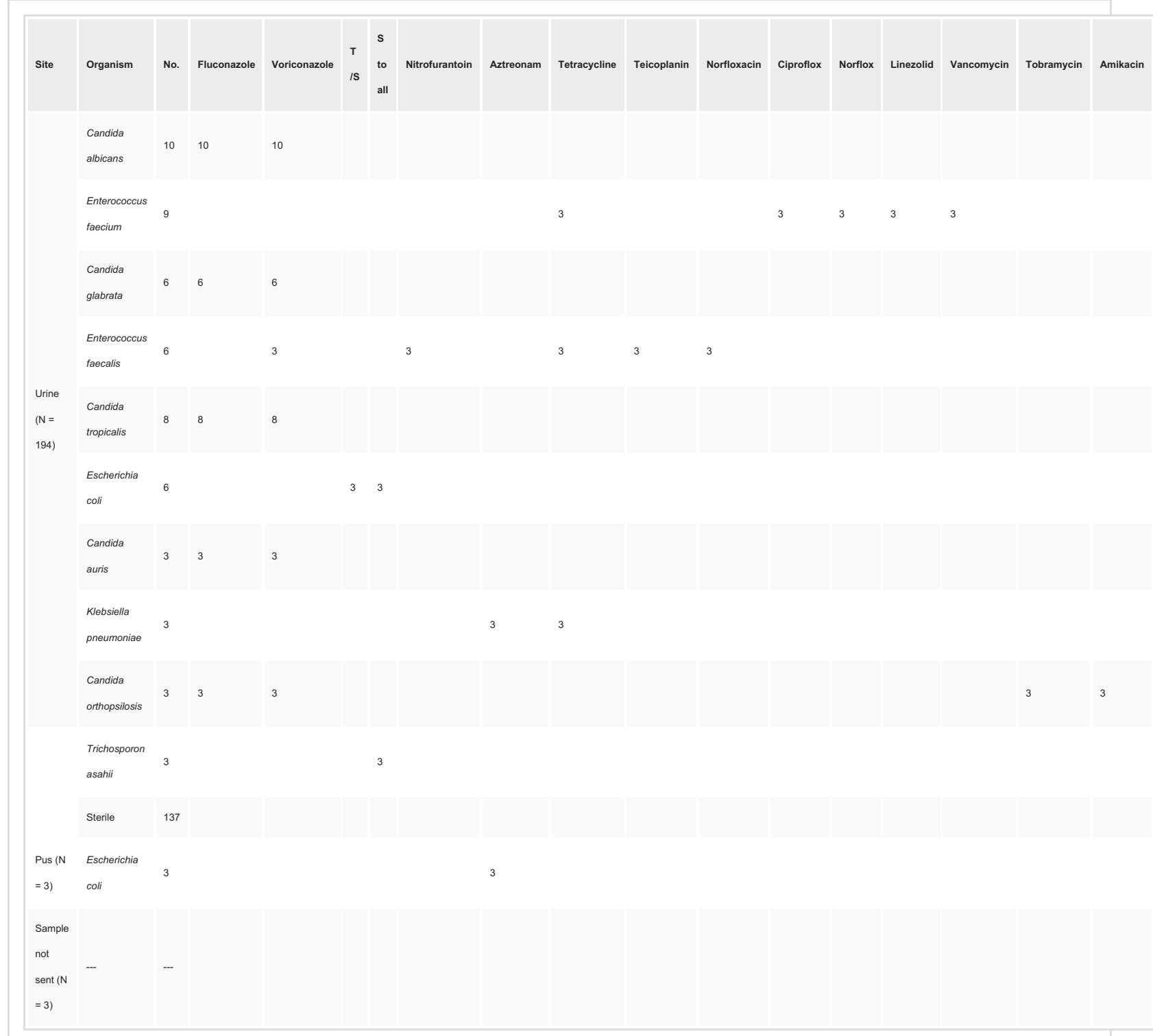

TABLE 4: Antibiotic susceptibility pattern of organisms in urine causing septicemia.

$S$ to all: sensitive to all primary antibiotics; T/S: trimethoprim/sulfamethoxazole

Of a total of 200 patients, 130 (65\%) were shifted to the parent unit, and 70 (35\%) patients died, with an ICU stay of $12.89 \pm 6.51$ days (Table 5).

\begin{tabular}{|c|c|c|}
\hline Variable & Number of patients $(\mathrm{N}=200)$ & Number of days in ICU \\
\hline Shifted to the referring parent unit & $130(65.0)$ & $13.73 \pm 6.94$ \\
\hline Expired & $70(35.0)$ & $11.32 \pm 5.29$ \\
\hline Total & & $12.89 \pm 6.51$ \\
\hline
\end{tabular}

TABLE 5: Clinical outcomes of ICU patients.

ICU: intensive care unit 


\section{Cureus}

of the 14 patients were transferred to the referring parent unit (Table 6).

\section{Variable}

Shifted to the parent unit

Expired

Total
Number of patients $(\mathrm{N}=186)$

$116(62.4)$

$70(37.6)$

186
Number of days in ICU

$13.93 \pm 6.95$

$11.32 \pm 5.29$

$12.95 \pm 6.49$

TABLE 6: Clinical outcomes in mechanically ventilated patients.

ICU: intensive care unit

There was no significant difference in mortality when microorganisms grew either in ET aspirates or urine compared with sterile samples (insignificant P-value), but if microorganisms grew in blood, mortality was as high as $56.8 \%$ compared with $30.6 \%$ (Table 7 ).

\begin{tabular}{|c|c|c|c|c|c|c|}
\hline \multirow{2}{*}{ Organism } & \multicolumn{2}{|c|}{ ET $(\mathrm{N}=186)$} & \multicolumn{2}{|c|}{ Urine $(\mathrm{N}=194)$} & \multicolumn{2}{|c|}{ Blood $(\mathrm{N}=197)$} \\
\hline & Number & Mortality & Number & Mortality & Number & Mortality \\
\hline Positive growth & 128 & $50(39.0 \%)$ & 57 & $20(35.1 \%)$ & 37 & $21(56.8 \%)$ \\
\hline Sterile & 58 & $20(34.4 \%)$ & 137 & $50(36.5 \%)$ & 160 & $49(30.6 \%)$ \\
\hline P-value & 0.550 & & 0.851 & & 0.002 & \\
\hline
\end{tabular}

TABLE 7: Mortality ratio for positive growth and sterile organisms on the basis of samples.

ET: endotracheal

If microorganisms growing in ET aspirates were resistant to all primary antibiotics, mortality was $60.6 \%$ (Table 8).

\begin{tabular}{|c|c|c|c|}
\hline Site & Organism & Sensitivity & Mortality \\
\hline ETA $(N=33)$ & Acinetobacter baumannii & Resistant to all antibiotics & $20(60.6 \%)$ \\
\hline
\end{tabular}

TABLE 8: Mortality in multidrug-resistant organisms in ETA.

ETA: endotracheal aspirate

\section{Discussion}

Sepsis is defined as life-threatening organ dysfunction caused by a dysregulated host response to infection [5]. The Surviving Sepsis Campaign recommends attempting to prove the presence of an infection by methods such as microbiological testing [6]. The gold standard to detect bloodstream infections is blood culture in a liquid media [7], followed by cultural and biochemical identification of the culprit pathogen. Thus, rapid blood culture testing with pathogen identification is essential to establish a diagnosis and enable efficient therapy.

Severe sepsis and septic shock are the most common causes of morbidity and mortality in critically ill patients. Angus et al. [7] reported severe sepsis incidence of 2.26 cases per 100 hospital discharges, with $51.1 \%$ requiring intensive care. The overall mortality rate was $28.6 \%$, which increased in patients with comorbidities. The overall mortality in gram-negative bacteremia is $25 \%$. When septic shock develops, the mortality increases to 50-60\% [8]. However, despite dramatic improvements in our knowledge of the pathogenesis, diagnosis, and therapeutic and supportive care, the mortality in septic patients remains unacceptably high, ranging from $30 \%$ to $50 \%$ in severe sepsis, and increasing to $50-87 \%$ in septic shock 
patients [9].

In the study, we found that the most common organism isolated from ET aspirates $(\mathrm{n}=186)$ was $A$. baumanii (91), followed by K. pneumoniae (14) and E. coli (9). In urine, out of 197 samples, the most common organisms were C. albicans (10) and E. faecium (9). In blood ( $\mathrm{n}=197)$, the most common organisms were $P$. aeuroginosa (10), K. pneumoniae (9), and A. baumanii (9). This study was conducted in a government-aided hospital where we received referred patients with sepsis-induced MODS with multidrug-resistant infections. Cross-infection can be a cause for the high incidence of multidrug-resistant organisms. As we included patients who developed a fever after admission that may be one of the causes of high incidence. Our results are consistent with the increasing trend of gram-negative septicemia in ICU [8]. Thus, there is a need for early diagnosis and species identification to initiate species-specific antibiotics and early shifting from empirical to a specific therapy. Traditional techniques for culture and antibiotic susceptibility usually take four to five days to provide results and delay the initiation of appropriate antibiotics. Our findings were comparable to the study by Khwannimit and Bhurayanontachai [9] who reported that the respiratory tract was the most common site for both community and hospital-acquired infections.

In our study, $10 \%$ of patients had diabetes, followed by hypertension (7.5\%), chronic obstructive pulmonary disorder (4.5\%), COVID-19 (3.5\%), and HCV (3.0\%), whereas 72\% did not have any of the above risk factors. Our findings were consistent with those of Zhong et al. [10] who reported diabetes mellitus, chronic obstructive pulmonary disease, and chronic hepatic insufficiency as the major risk factors for bacterial bloodstream infections. About $72 \%$ of our patients did not have any comorbidity; therefore, the data are inconclusive to draw results.

A major advantage of using MALDI-TOF MS was that it reduced the time between blood drawing and receiving the results about organisms causing sepsis [11]. Our findings were consistent with the previous study proving the efficacy of MALDI-TOF MS in critically ill patients [3]. We collected hospital data from our ICU to see any mortality benefit. In 2018, mortality was $35.36 \%$ (325/919), and in 2019, it was $35.34 \%$ (328/928). Thus, in our study, it was about 35\%, although mortality is multifactorial and does not depend on one factor. Hence, we cannot conclude that MALDI-TOF MS has mortality benefits in sepsis patients, but it has an established role in early detection, early initiation of specific antibiotics, early de-escalation of antibiotics, and reduced ICU stay.

Considering the antibiotics susceptibility of the organisms isolated from ET aspirates, A. baumanii was most susceptible to tetracycline, tobramycin, levofloxacin, and meropenem. In blood, $P$. aeuroginosa was the most common isolated organism and was susceptible to piperacillin-tazobactam and gentamycin. In urine, isolated Candida spp. was susceptible to both fluconazole and voriconazole. Patients who developed symptoms or neutropenia were treated with fluconazole.

We observed clinical outcomes of the patients regarding ICU stay. Of a total of 200 patients, 130 (65\%) patients shifted to the referring parent unit, and 70 (35\%) patients died, with an ICU stay of $12.89 \pm 6.51$ days. There was no significant difference in mortality when microorganisms grew either in ET or urine compared with sterile samples. If microorganisms resistant to all primary antibiotics grew from ET, mortality was $60.6 \%$. Mortality was $56.8 \%$ if the isolates were in the blood. Out of 200 patients, 186 patients were mechanically ventilated, with mortality of $37 \%$, and the rest 14 patients were transferred to the parent referring unit. Therefore, mechanical ventilation is an independent risk factor of mortality in ICU. Zhong et al. [10] reported that in 117 patients, the mean ICU stay was 14 days. Further 28-day mortality was 35.0\%, whereas 60 -day mortality was $39.3 \%$, and in-hospital mortality was $42.7 \%$. Su et al. [1] also reported on the clinical impact of patients with bloodstream infection with different groups of viridians streptococci by using MALDI-TOF MS, concluding that the mean ICU stay was significantly higher in patients who survived than those who died $(\mathrm{P}<0.01)$.

The present study has some limitations. Because it was a single-center study of one ICU, antibiogram and organisms may not reflect the actual burden. Moreover, there was no data to compare the results of MALDITOF MS with the traditional methods. Due to the SARS-CoV-2 infection, results became incomparable to the past data. The sample size was small to demonstrate any mortality benefit of MALDI-TOF MS and the inability to discriminate between related species. Additionally, MALDI-TOF MS is currently unable to differentiate E. coli from Shigella.

\section{Conclusions}

As the incidence of gram-negative organisms is rapidly increasing in sepsis patients, early recognition and species identification can be achieved by MALDI-TOF MS, and initiation of appropriate antibiotics plays an important role in the treatment and care of patients. MALDI-TOF MS has the potential to revolutionize sepsis management. A major advantage of using MALDI-TOF MS was that it reduced the time between blood drawing and receiving the results regarding organisms causing sepsis. Although the time required for the identification by MALDI-TOF MS is 10 minutes/strain, we received the final sensitivity reports within 48 hours, which earlier used to take four to five days. MALDI-TOF MS is quick and accurate, providing species identification within two to six hours from positive growth to sensitivity testing and reporting. Our findings show that the use of MALDI-TOF MS has the potential to replace traditional methods of species 
identification, and routine use may have mortality benefits in patients with sepsis as sepsis management is time-specific. The tool has a role in the early initiation of specific therapy and early de-escalation of treatment. A larger randomized control trial is needed to establish mortality benefits.

\section{Additional Information \\ Disclosures}

Human subjects: Consent was obtained or waived by all participants in this study. Institutional Ethics Committee (King George's Medical University, Lucknow, India) issued approval 102 ECM II B-THESIS/P79. Animal subjects: All authors have confirmed that this study did not involve animal subjects or tissue. Conflicts of interest: In compliance with the ICMJE uniform disclosure form, all authors declare the following: Payment/services info: All authors have declared that no financial support was received from any organization for the submitted work. Financial relationships: All authors have declared that they have no financial relationships at present or within the previous three years with any organizations that might have an interest in the submitted work. Other relationships: All authors have declared that there are no other relationships or activities that could appear to have influenced the submitted work.

\section{References}

1. Dellinger RP, Levy MM, Carlet JM, et al.: Surviving Sepsis Campaign: international guidelines for management of severe sepsis and septic shock: 2008. Crit Care Med. 2008, 36:296-327. 10.1097/01.CCM.0000298158.12101.41

2. Washington JA 2nd, Ilstrup DM: Blood cultures: issues and controversies. Rev Infect Dis. 1986, 8:792-802. 10.1093/clinids/8.5.792

3. Schubert S, Kostrzewa M: MALDI-TOF MS in the microbiology laboratory: current trends. Curr Issues Mol Biol. 2017, 23:17-20. 10.21775/cimb.023.017

4. Chatterjee S, Bhattacharya M, Todi SK: Epidemiology of adult-population sepsis in India: a single center 5 year experience. Indian J Crit Care Med. 2017, 21:573-7. 10.4103/ijccm.IJCCM_240_17

5. Napolitano LM: Sepsis 2018: definitions and guideline changes. Surg Infect (Larchmt). 2018, 19:117-25. 10.1089/sur.2017.278

6. Kumar A, Roberts D, Wood KE, et al.: Duration of hypotension before initiation of effective antimicrobial therapy is the critical determinant of survival in human septic shock. Crit Care Med. 2006, 34:1589-96. 10.1097/01.CCM.0000217961.75225.E9

7. Angus DC, Linde-Zwirble WT, Lidicker J, Clermont G, Carcillo J, Pinsky MR: Epidemiology of severe sepsis in the United States: analysis of incidence, outcome, and associated costs of care. Crit Care Med. 2001, 29:1303-10. 10.1097/00003246-200107000-00002

8. Vincent JL, Sakr Y, Sprung CL, et al.: Sepsis in European intensive care units: results of the SOAP study . Crit Care Med. 2006, 34:344-53. 10.1097/01.ccm.0000194725.48928.3a

9. Khwannimit B, Bhurayanontachai R: The epidemiology of, and risk factors for, mortality from severe sepsis and septic shock in a tertiary-care university hospital setting. Epidemiol Infect. 2009, 137:1333-41. 10.1017/S0950268809002027

10. Zhong L, Zhang S, Tang K, et al.: Clinical characteristics, risk factors and outcomes of mixed Candida albicans/bacterial bloodstream infections. BMC Infect Dis. 2020, 20:810. 10.1186/s12879-020-05536-Z

11. Homolová R, Bogdanová K, Bardoň J, Kolář M: [Direct identification of bacteria in blood cultures by MALDITOF MS]. Klin Mikrobiol Infekc Lek. 2020, 26:45-50. 